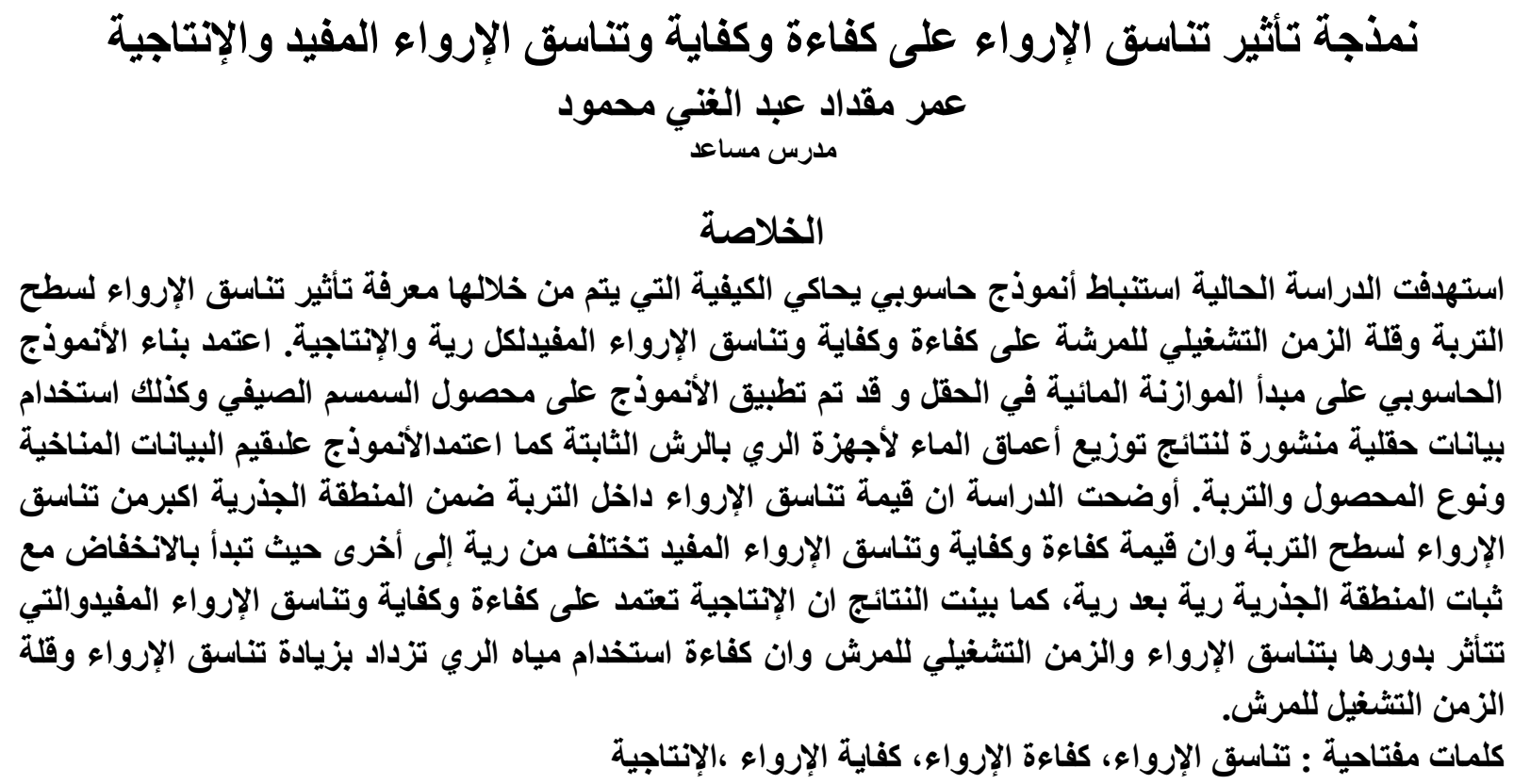

\title{
Modeling The Impact of Application Uniformity on The Efficiency ,Adequacy ,Useful Application Uniformity And Productivity
}

\section{OMAR MUQDAD ABDULGANY MAHMOOD}

\begin{abstract}
The study aims to develop computer model that simulates how a specimen by which to know the impact of Application uniformity on the soil surface and the lack of operating time for the sprinkler on the efficiency, adequacy, useful application uniformity of each irrigation and productivity. The model building adopted on the principle of water balance in the field and has been applied to sesame summer crop by using available field data for the water distribution under sprinkler system. The model also adopted the climate data and the type of crop and soil. The study show that useful uniformity coefficient more than uniformity on the soil surface and the value of the efficiency and adequacy and useful uniformity vary from one irrigation to another and start to decline with the stability of the root zone irrigation after irrigation, and the results show that productivity depends on the efficiency, adequacy and useful uniformity that are affected in turn byuniformity and the time of application. the water use efficiency increased by increasing the application uniformity and decreasing the sprinkler operating time.
\end{abstract}




\section{المقدمة}

إن متابعة ما يحصل داخل التربة يومياً لمحصول ما ولعدة مواقع في الحقل خلال الموسم، وربط ذلك مع معدل نمو

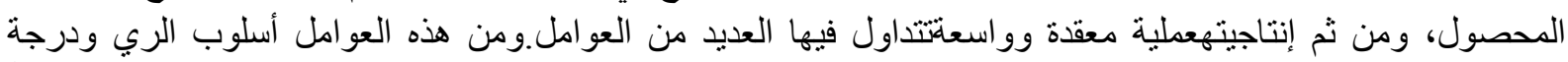

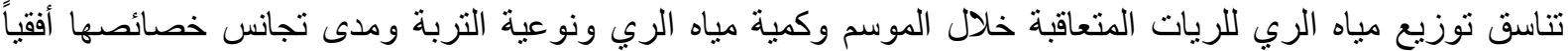

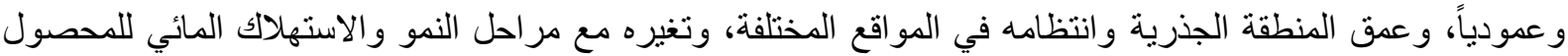

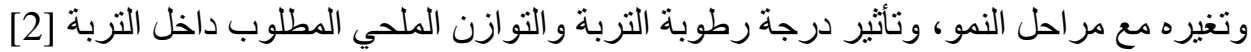

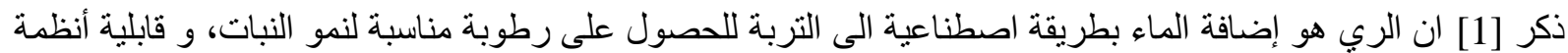

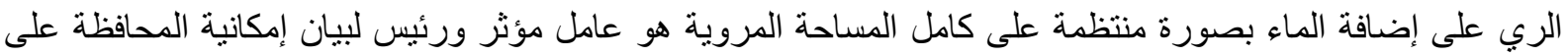

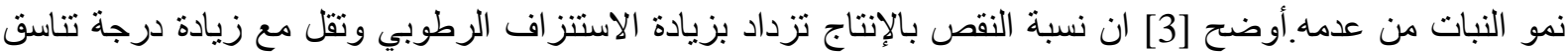

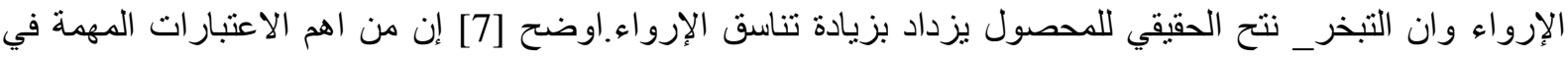

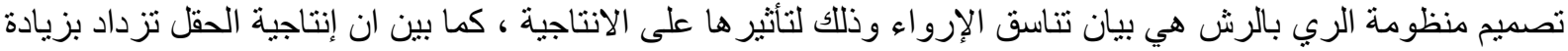

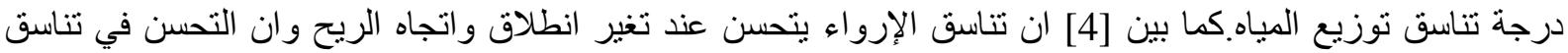

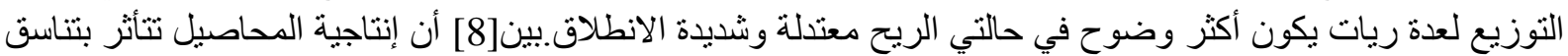

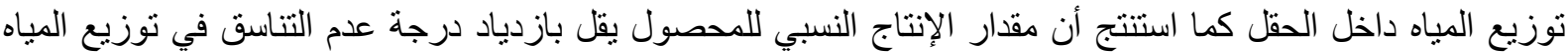

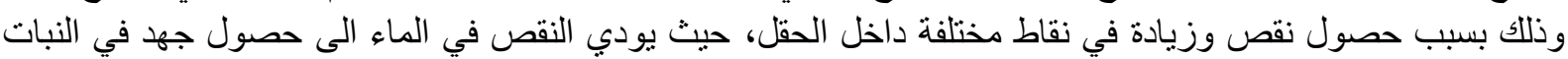

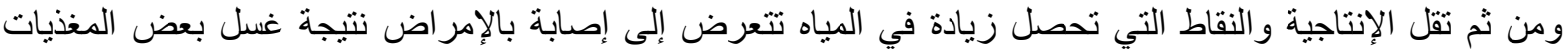

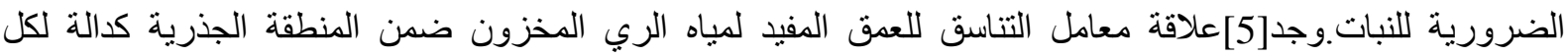

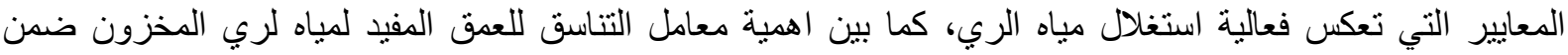
المنطقة الجذرية وذلك عند استخدام ريات ذات توزيع ثابت وبتغيير كفاية الارواء من خلال التهائ التحكم بفاصلة الارواء.

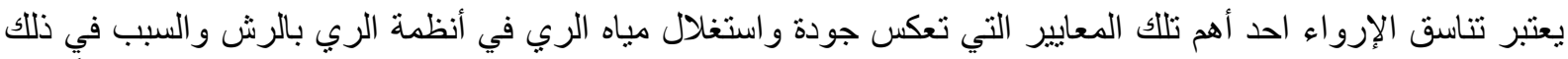

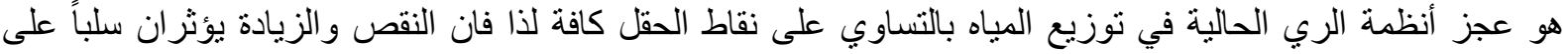

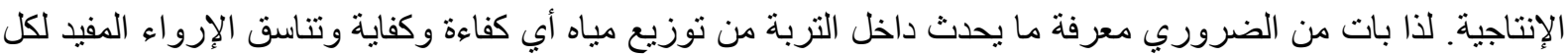

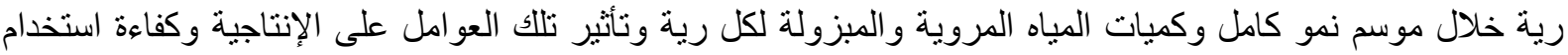
مياه الري.

طريقة البحث

تم بناء الانموذج الحاسوبي باستخدام ماتلابحيث يتم ادخال معلومات عن نوع التربة والمحصول و البيانات المناخية اليومية

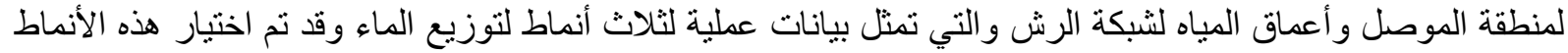

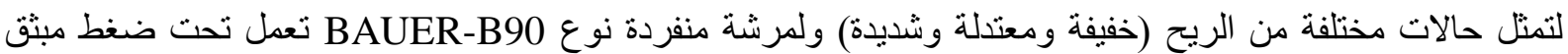

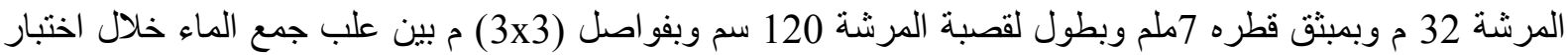

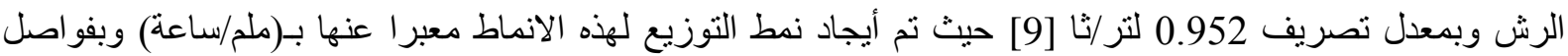

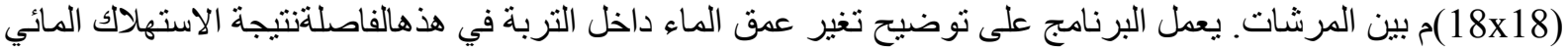

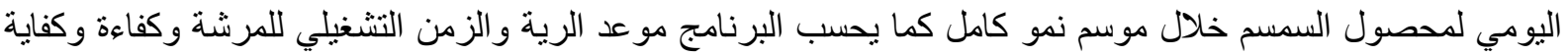

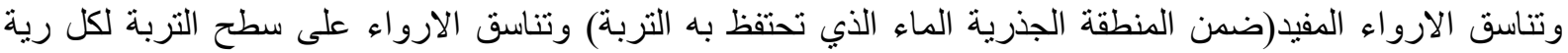
وكمية المياه المضافة و المبزولة لكل رية. وقد تم اعتماد ذلك باعتماد الخطوات التئة التالية:

1- يتم تحديد نمط الإرواء المستخدم (p1,p2,p3] نمط واحد لموسم نمو كامل ومن ثم يتم إيجاد معامل تناسق توزيع الماء باستخدام معامل كرستننسن.

$U c c=\left(1-\frac{\sum_{1}^{n}\left|\left(x_{i}-\bar{x}\right)\right|}{n \bar{x}}\right) \times 100$ 


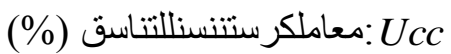

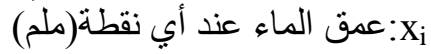

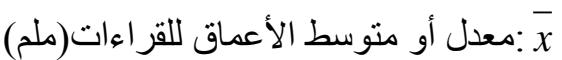

: عدنقاط القياس :

2- يتم تحديد النسبة المئوية للزمن التشغيلي للمرشة من الزمن اللازم لإعطاء معدل اعماق المياه الى السعة الحقلية OT $\%(100 ، 80 ، 60 ، 40)$

3- حساب الاستهلاك المائي للمحصول لكل نقطة 36 ضمن شبكة الري (18x18) م باستخدام معادلة الـ56 FAO

$E T_{C a}=K_{c} \times K_{s} \times E T_{c}$

:الاستهلاك المائي للمحصول (ملم/يوم). $E T_{c a}$

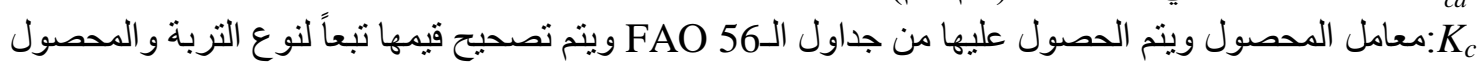
و الظروف المناخية. :معامل جهد ماء التربة (يعتمد على نسبة الاستنز اف و الماء المتيسر الكلي و الماء المتيسر بسهولة) ET :

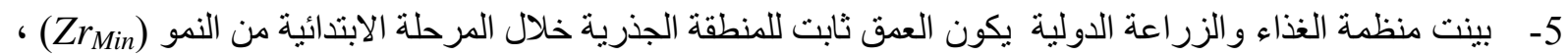

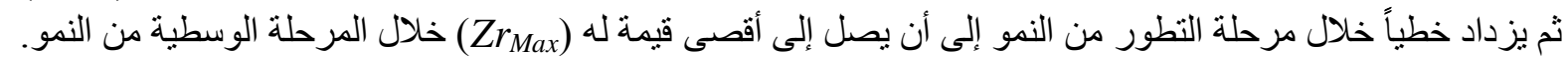
$Z r=Z r_{\text {Min }}+\left(Z r_{\text {Max }}-Z r_{\text {Min }}\right) \frac{J-J_{\text {start }}}{J_{\text {Max }}-J_{\text {start }}}$

Z Z $r_{\text {Min }}$

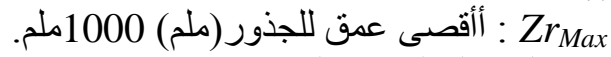

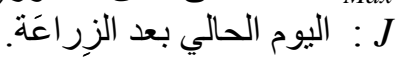

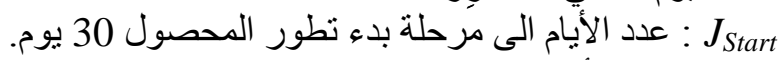
J $J_{\text {Max }}$

6- يتم تحديد موعد الرية عندما يصل معدل الاستنزاف الرطوبي 65\% من الماء المتيسر الكلي ، عندها يتم الإرواء وفق النمط المستخدم وكذلك تحديد زمن الإرواء و عمق مياه الري لكل نقطة .

7- يتم حساب كفاءة وكفايةالارو اء لكل رية من خلال المعادلات التالية.

$A \%=\left(\frac{N_{O}}{N_{T}}\right) * 100 \%$ $-4$

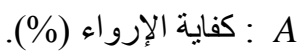
No $N_{0}$ NT

$E \%=(100 \%-S S L) \times(100 \%-D P L)$ $-5$

E : كفاءة الإرواء (\%) : E : فو اقد رذاذ الرش \% : SSL DPL 


$$
S S L \%=\left(\frac{V_{S P R I N K L E R}-V_{\text {SOIL }}}{V_{\text {SPRINKLER }}}\right) \times 100
$$

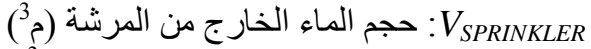

VOIL

$D P L \%=\left(\frac{D R}{I R}\right) \times 100 \%$

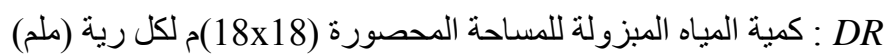

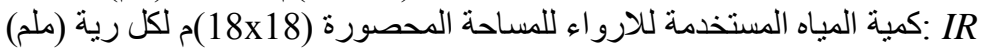
8- يتم حساب معامل تتاسق توزيع الماء المفيد لكل رية من خلال إيجاد عمق الماء المفيد داخل التربة في جميع المو اقع. 9- يتم حساب الانتاج النسبي وكفاء استخدام المياه خلال موسم نمو كامل من خلال معادلات FAO56.

$\left(\frac{Y_{m}-Y_{a}}{Y_{m}}\right)=K_{Y}\left(\frac{E T_{c}-E T_{c a}}{E T_{c}}\right)$ $-8$

Y : إنتاجية المحصول الفعلية (كغم). K : $Y_{m}$ $W U E=\frac{Y_{a}}{I r}$

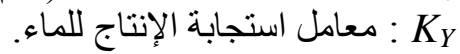

1- تناسق الإرواء : من خلال النتائج التي تم الحصول عليها الجدول من (1) إلى (12) نلاحظ بان تناسق الإرواء الإن المفيد

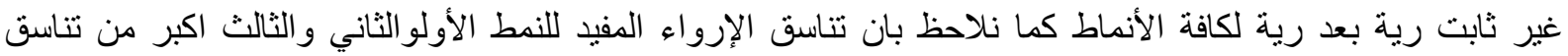

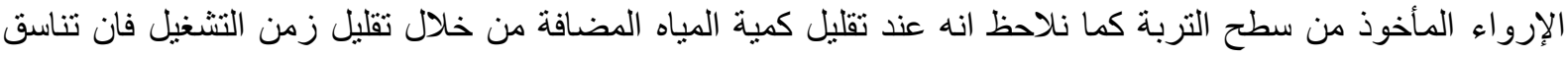

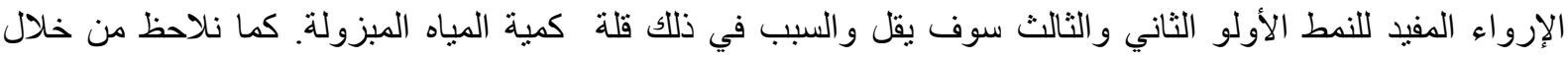

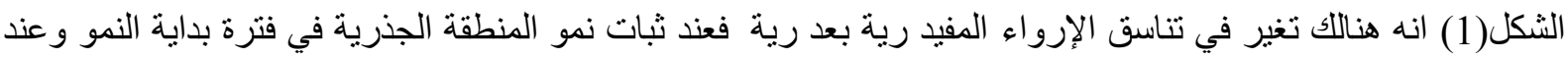

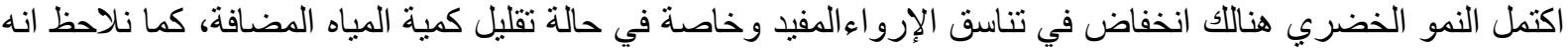

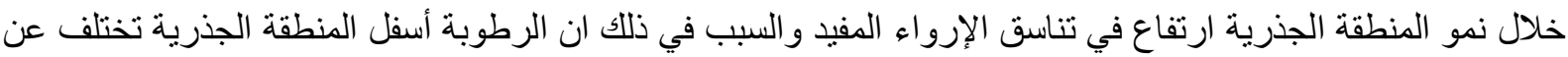

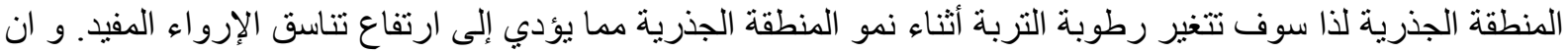

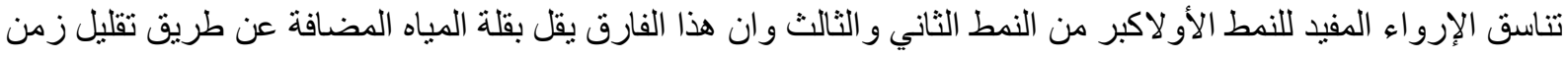

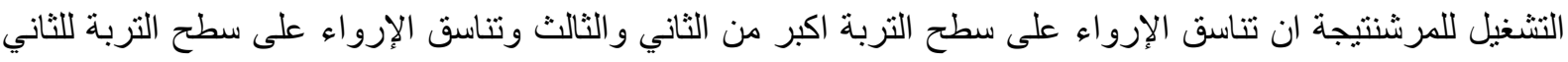
اكبر من الثالث.ونلاحظ من خلال الثكل(1) ان تناسق الإرواء المفيد خلال الموسم يكون على شكل حزمة لكافة الأنماط

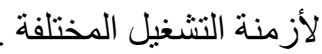

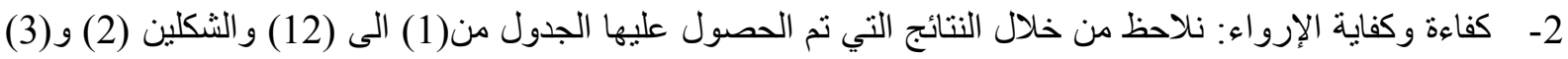

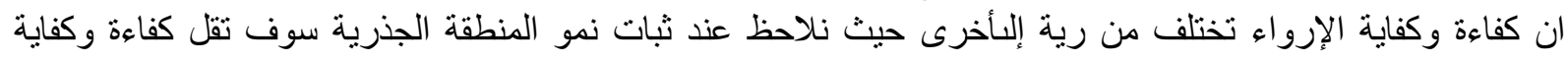

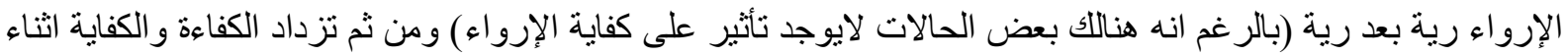

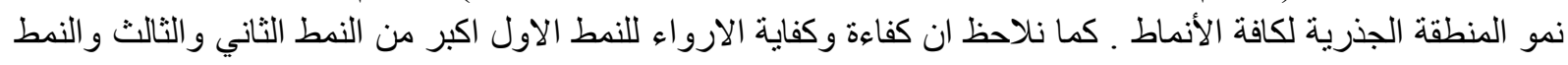


الثاني اكبر من الثالث نتيجة ان تناسق الارواء المفيد اكبر من الثاني والثالث وتناسق الارواء على سطح التربة للثاني اكبر

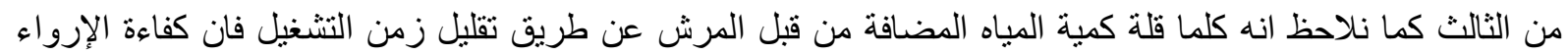

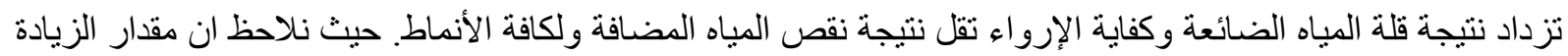

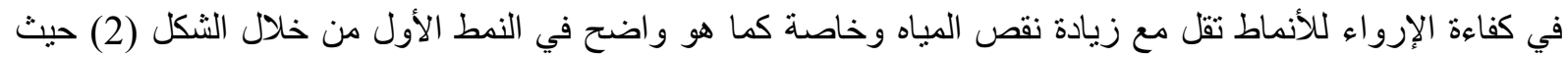

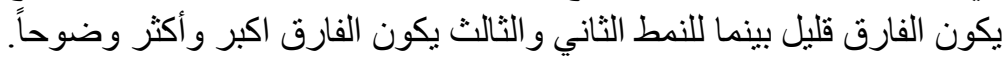

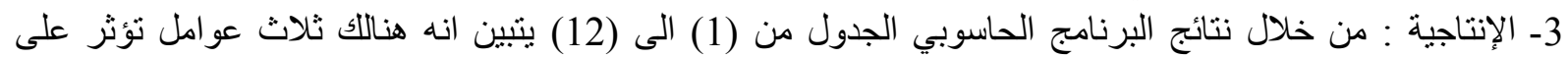

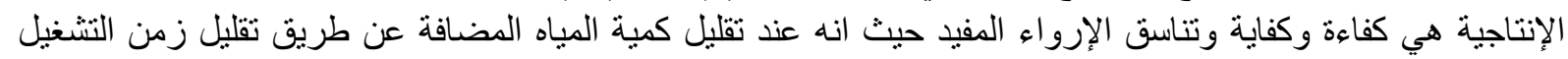

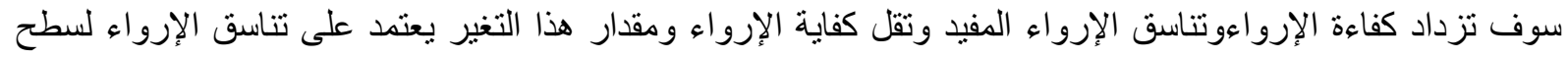

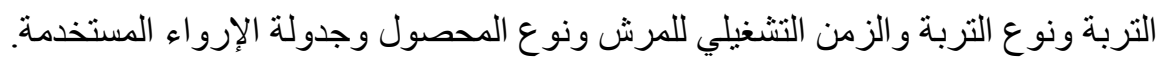

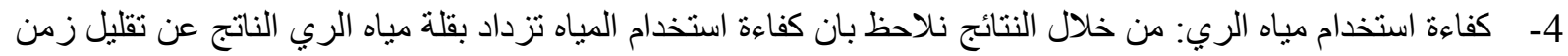

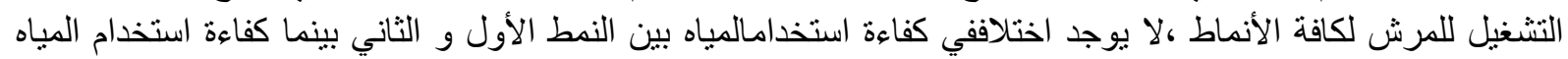

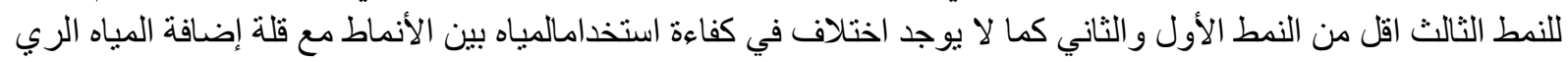
أي نستتنج بان كفاءة استخدام المياه تقل بقلة كفاءة الإرواء ولا تتأثر بقلة تناسق الإرواء في حالة الأزمنة التشغيلية القليلة.

الجدول (1) : نتائج الأنموذج الحاسوبي للنمط الأول ولزمن تشغيلي 100\%

\begin{tabular}{|c|c|c|c|c|c|c|c|c|c|c|}
\hline استخدام مياه & الحققيقي & نالإنتاجة & مبزولة & كمية المياه & الإرواية & الإرواء & الإرواء ألتاء & الإرواء & اليوم & التشغيل \\
\hline \multirow{11}{*}{0.15} & \multirow{11}{*}{773} & \multirow{11}{*}{0.97} & 45 & 710 & 1 & 0.79 & 0.97 & 0.87 & 7 & 133 \\
\hline & & & 66 & 735 & 1 & 0.77 & 0.95 & 0.87 & 14 & 138 \\
\hline & & & 73 & 739 & 1 & 0.76 & 0.95 & 0.87 & 21 & 138 \\
\hline & & & 76 & 759 & 1 & 0.76 & 0.95 & 0.87 & 28 & 142 \\
\hline & & & 129 & 1644 & 1 & 0.78 & 0.96 & 0.87 & 40 & 308 \\
\hline & & & 216 & 2922 & 1 & 0.79 & 0.96 & 0.87 & 53 & 548 \\
\hline & & & 288 & 3554 & 1 & 0.78 & 0.96 & 0.87 & 66 & 666 \\
\hline & & & 317 & 3556 & 1 & 0.77 & 0.95 & 0.87 & 80 & 666 \\
\hline & & & 342 & 3530 & 1 & 0.77 & 0.95 & 0.87 & 96 & 662 \\
\hline & & & 1552 & $\begin{array}{c}1814 \\
9\end{array}$ & \multicolumn{6}{|c|}{ المجموع } \\
\hline & & & 43 & 504 & \multicolumn{6}{|c|}{ المعدل لموسم نمو كامل } \\
\hline
\end{tabular}

الجدول (2) : نتائج الأنموذج الحاسوبي للنمط الأول ولزمن تشغيلي 80\%

\begin{tabular}{|c|c|c|c|c|c|c|c|c|c|c|}
\hline مياه الرداءم & الحققيقي & الإنتاجة & مبزولة & 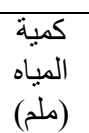 & الإرواية & الإرواءة & الإرواء & الإرواء & اليوم & التنتغيل \\
\hline \multirow{12}{*}{0.17} & \multirow{12}{*}{761} & \multirow{12}{*}{0.95} & 1 & 568 & 1 & 0.85 & 0.97 & 0.87 & 7 & 107 \\
\hline & & & 17 & 585 & 1 & 0.82 & 0.94 & 0.87 & 13 & 110 \\
\hline & & & 29 & 589 & 1 & 0.81 & 0.92 & $\begin{array}{l}0.87 \\
\end{array}$ & 19 & 110 \\
\hline & & & 32 & 613 & 1 & 0.80 & 0.92 & 0.87 & 25 & 115 \\
\hline & & & 34 & 719 & 1 & 0.81 & 0.92 & 0.87 & 32 & 135 \\
\hline & & & 18 & 1675 & 1 & 0.84 & 0.94 & $\begin{array}{c}0.87 \\
\end{array}$ & 44 & 314 \\
\hline & & & 35 & 2538 & 1 & 0.84 & 0.95 & 0.87 & 56 & 476 \\
\hline & & & 69 & 2942 & 1 & 0.83 & 0.94 & 0.87 & 68 & 551 \\
\hline & & & 114 & 2842 & 1 & 0.81 & 0.93 & 0.87 & 80 & 533 \\
\hline & & & 136 & 2831 & 1 & 0.81 & 0.93 & $\begin{array}{l}0.87 \\
\end{array}$ & 93 & 531 \\
\hline & & & 485 & $\begin{array}{c}1590 \\
1\end{array}$ & \multicolumn{6}{|c|}{ المجموع } \\
\hline & & & 13 & 442 & \multicolumn{6}{|c|}{ المعدل لموسم نمو كامل } \\
\hline
\end{tabular}


الجدول (3) : نتائج الأنموذج الحاسوبي للنمط الأول ولزمن تشغيلي 60\%

\begin{tabular}{|c|c|c|c|c|c|c|c|c|c|c|}
\hline كباه الرئخدام & الحقيقي & الإنتاج & لة المياهمبزو & كميةالم & الإرواءة & الإرواء كفاءة & الإرواء & الإرواء & اليوم & (دقنشيل \\
\hline \multirow{15}{*}{0.17} & \multirow{15}{*}{747} & \multirow{15}{*}{0.93} & 0 & 426 & 1.00 & 0.85 & 0.97 & 0.87 & 7 & 80 \\
\hline & & & 0 & 467 & 1.00 & 0.85 & 0.94 & 0.87 & 12 & 87 \\
\hline & & & 3 & 472 & 0.92 & 0.84 & 0.92 & 0.87 & 17 & 88 \\
\hline & & & 12 & 422 & 0.89 & 0.82 & 0.91 & 0.87 & 21 & 79 \\
\hline & & & 8 & 464 & 0.89 & 0.83 & 0.89 & 0.87 & 26 & 87 \\
\hline & & & 19 & 423 & 0.89 & 0.81 & 0.89 & 0.87 & 30 & 79 \\
\hline & & & 0 & 994 & 1.00 & 0.85 & 0.93 & 0.87 & 40 & 186 \\
\hline & & & 0 & 1609 & 1.00 & 0.85 & 0.94 & 0.87 & 50 & 302 \\
\hline & & & 0 & 2209 & 1.00 & 0.85 & 0.95 & 0.87 & 61 & 414 \\
\hline & & & 13 & 2166 & 1.00 & 0.84 & 0.93 & 0.87 & 70 & 406 \\
\hline & & & 21 & 2202 & 1.00 & 0.84 & 0.93 & 0.87 & 80 & 413 \\
\hline & & & 41 & 2166 & 1.00 & 0.83 & 0.92 & 0.87 & 90 & 406 \\
\hline & & & 58 & 2128 & 0.97 & 0.82 & 0.91 & 0.87 & 106 & 399 \\
\hline & & & 175 & 16148 & \multicolumn{6}{|c|}{ المجموع } \\
\hline & & & 5 & 449 & \multicolumn{6}{|c|}{ المعدل لموسم نمو كامل } \\
\hline
\end{tabular}

الجدول (4) : نتائج الأنموذج الحاسوبي للنمط الأول ولزمن تشغيلي 40\%

\begin{tabular}{|c|c|c|c|c|c|c|c|c|c|c|}
\hline استخداءم & الإقتاجي & الإنتاج & بزولة المياهد & كمية كلة & كفاية & الإرواء كفاءة & الإرواء & الإرواء & اليوم & زلتشنيل \\
\hline \multirow{20}{*}{0.18} & \multirow{20}{*}{738} & \multirow{20}{*}{0.92} & 0 & 284 & 1.00 & 0.85 & 0.98 & 0.87 & 7 & 53 \\
\hline & & & 0 & 291 & 1.00 & 0.85 & 0.96 & 0.87 & 10 & 55 \\
\hline & & & 0 & 289 & 0.89 & 0.85 & 0.94 & 0.87 & 13 & 54 \\
\hline & & & 0 & 291 & 0.89 & 0.85 & 0.92 & 0.87 & 16 & 55 \\
\hline & & & 0 & 285 & 0.89 & 0.85 & 0.91 & 0.87 & 19 & 53 \\
\hline & & & 2 & 287 & 0.89 & 0.84 & 0.90 & 0.87 & 22 & 54 \\
\hline & & & 3 & 292 & 0.89 & 0.84 & 0.89 & 0.87 & 25 & 55 \\
\hline & & & 4 & 289 & 0.89 & 0.84 & 0.88 & 0.87 & 28 & 54 \\
\hline & & & 0 & 433 & 1.00 & 0.85 & 0.91 & 0.87 & 34 & 81 \\
\hline & & & 0 & 761 & 1.00 & 0.85 & 0.94 & 0.87 & 42 & 143 \\
\hline & & & 0 & 1016 & 1.00 & 0.85 & 0.95 & 0.87 & 49 & 190 \\
\hline & & & 0 & 1261 & 1.00 & 0.85 & 0.95 & 0.87 & 56 & 236 \\
\hline & & & 0 & 1446 & 1.00 & 0.85 & 0.95 & 0.87 & 63 & 271 \\
\hline & & & 0 & 1427 & 1.00 & 0.85 & 0.94 & 0.87 & 69 & 268 \\
\hline & & & 0 & 1463 & 0.92 & 0.85 & 0.94 & 0.87 & 76 & 274 \\
\hline & & & 0 & 1474 & 0.92 & 0.85 & 0.93 & 0.87 & 83 & 276 \\
\hline & & & 3 & 1448 & 0.92 & 0.85 & 0.93 & 0.87 & 90 & 271 \\
\hline & & & 22 & 1410 & 0.89 & 0.83 & 0.92 & 0.87 & 99 & 264 \\
\hline & & & 34 & $\begin{array}{c}1444 \\
9\end{array}$ & \multicolumn{6}{|c|}{ المجموع } \\
\hline & & & 1 & 401 & \multicolumn{6}{|c|}{ المعدل لموسم نمو كامل } \\
\hline
\end{tabular}


محمود: نمذجة تأثير تناسق الإرواء على كفاءة وكفاية وتناست الإرواء المفيد والإنتاجية

الجدول (5) : نتائج الأنموذج الحاسوبي للنمط الثاني ولزمن تشغيلي 100\%

\begin{tabular}{|c|c|c|c|c|c|c|c|c|c|c|}
\hline كيتخدام & الحقيقي & الإنتاج & مبزولة & كمية & الإرواءء & الإرواءة & الإرواء & الإرواء & 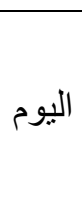 & زالتشغيل \\
\hline \multirow{11}{*}{0.15} & \multirow{11}{*}{762} & \multirow{11}{*}{0.95} & 75 & 710 & 1.00 & 0.70 & 0.96 & 0.79 & 7 & 144 \\
\hline & & & 117 & 762 & 1.00 & 0.66 & 0.93 & 0.79 & 14 & 155 \\
\hline & & & 131 & 777 & 1.00 & 0.65 & 0.91 & 0.79 & 21 & 158 \\
\hline & & & 137 & 714 & 1.00 & 0.63 & 0.91 & 0.79 & 27 & 145 \\
\hline & & & 210 & 1454 & 1.00 & 0.67 & 0.93 & 0.79 & 38 & 296 \\
\hline & & & 347 & 2611 & 1.00 & 0.67 & 0.94 & 0.79 & 50 & 531 \\
\hline & & & 495 & 3578 & 1.00 & 0.67 & 0.94 & 0.79 & 63 & 727 \\
\hline & & & 555 & 3581 & 1.00 & 0.66 & 0.93 & 0.79 & 76 & 728 \\
\hline & & & 581 & 3641 & 1.00 & 0.65 & 0.92 & 0.79 & 90 & 740 \\
\hline & & & 2648 & $\begin{array}{c}1783 \\
0\end{array}$ & \multicolumn{6}{|c|}{ المجموع } \\
\hline & & & 74 & 495 & \multicolumn{6}{|c|}{ المعدل لموسم نمو كامل } \\
\hline
\end{tabular}

الجدول (6) : نتائج الأنموذج الحاسوبي للنمط الثاني ولزمن نشغيلي 80\%

\begin{tabular}{|c|c|c|c|c|c|c|c|c|c|c|}
\hline استخداءة & الإِتَّي & الإنتاج & لز بزولة & كميةًا & الإرواء كفاية & الإرواءة & الإرواء & الإرواء & 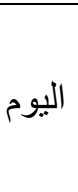 & التشغيل \\
\hline \multirow{12}{*}{0.17} & \multirow{12}{*}{744} & \multirow{12}{*}{0.93} & 8 & 568 & 1.00 & 0.77 & 0.94 & 0.79 & 7 & 116 \\
\hline & & & 47 & 588 & 1.00 & 0.72 & 0.91 & 0.79 & 13 & 119 \\
\hline & & & 62 & 602 & 1.00 & 0.70 & 0.89 & 0.79 & 19 & 122 \\
\hline & & & 65 & 622 & 1.00 & 0.70 & 0.88 & 0.79 & 25 & 126 \\
\hline & & & 67 & 725 & 1.00 & 0.71 & 0.89 & 0.79 & 32 & 147 \\
\hline & & & 76 & 1539 & 1.00 & 0.74 & 0.92 & 0.79 & 43 & 313 \\
\hline & & & 110 & 2480 & 1.00 & 0.74 & 0.92 & 0.79 & 55 & 504 \\
\hline & & & 192 & 2815 & 1.00 & 0.73 & 0.91 & 0.79 & 66 & 572 \\
\hline & & & 243 & 2845 & 1.00 & 0.71 & 0.90 & 0.79 & 78 & 578 \\
\hline & & & 278 & 2815 & 1.00 & 0.70 & 0.90 & 0.79 & 90 & 572 \\
\hline & & & 1148 & $\begin{array}{c}1560 \\
0\end{array}$ & \multicolumn{6}{|c|}{ المجموع } \\
\hline & & & 32 & 433 & \multicolumn{6}{|c|}{ المعدل لموسم نمو كامل } \\
\hline
\end{tabular}


الجدول (7) : نتائج الأنموذج الحاسوبي للنمط الثاني ولزمن تشغيلي 60\%

\begin{tabular}{|c|c|c|c|c|c|c|c|c|c|c|}
\hline استخداءم & الحقيقي & الإنتاج & (ملياهد & كميّة & الإرواء كفاية & كفاءة الإرواء & الإرواء & الإرواء & اليوم اليوم & زالتشغيل \\
\hline \multirow{14}{*}{0.17} & \multirow{14}{*}{723} & \multirow{14}{*}{0.90} & 0 & 426 & 1.00 & 0.78 & 0.95 & 0.79 & 7 & 87 \\
\hline & & & 0 & 465 & 1.00 & 0.78 & 0.90 & 0.79 & 12 & 94 \\
\hline & & & 17 & 462 & 0.86 & 0.75 & 0.87 & 0.79 & 17 & 94 \\
\hline & & & 24 & 464 & 0.86 & 0.74 & 0.86 & 0.79 & 22 & 94 \\
\hline & & & 40 & 426 & 0.83 & 0.70 & 0.86 & 0.79 & 26 & 87 \\
\hline & & & 16 & 542 & 1.00 & 0.76 & 0.87 & 0.79 & 32 & 110 \\
\hline & & & 0 & 1114 & 1.00 & 0.78 & 0.90 & 0.79 & 42 & 226 \\
\hline & & & 0 & 1699 & 1.00 & 0.78 & 0.91 & 0.79 & 52 & 345 \\
\hline & & & 13 & 2120 & 1.00 & 0.77 & 0.91 & 0.79 & 62 & 431 \\
\hline & & & 56 & 2201 & 1.00 & 0.76 & 0.89 & 0.79 & 72 & 447 \\
\hline & & & 84 & 2176 & 1.00 & 0.75 & 0.89 & 0.79 & 82 & 442 \\
\hline & & & 106 & 2172 & 0.97 & 0.74 & 0.88 & 0.79 & 93 & 441 \\
\hline & & & 357 & $\begin{array}{c}1426 \\
7\end{array}$ & \multicolumn{6}{|c|}{ المجموع } \\
\hline & & & 10 & 396 & \multicolumn{6}{|c|}{ المعدل لموسم نمو كامل } \\
\hline
\end{tabular}

الجدول (8) : نتائج الأنموذج الحاسوبي للنمط الثاني ولزمن تشغيلي 40\%

\begin{tabular}{|c|c|c|c|c|c|c|c|c|c|c|}
\hline مياه الريدام & الالإنتاجي & الإنتاجة & ولة المياهمبز ( & كياهة| كمئة & الإرواءية & الإرواءة & الإرباء & تالناسق & اليوم & التشتغيل \\
\hline \multirow{20}{*}{0.18} & \multirow{20}{*}{723} & \multirow{20}{*}{0.89} & 0 & 284 & 1.00 & 0.78 & 0.97 & 0.79 & 7 & 58 \\
\hline & & & 0 & 291 & 1.00 & 0.78 & 0.93 & 0.79 & 10 & 59 \\
\hline & & & 0 & 288 & 0.83 & 0.78 & 0.90 & 0.79 & 13 & 59 \\
\hline & & & 0 & 287 & 0.81 & 0.78 & 0.87 & 0.79 & 16 & 58 \\
\hline & & & 0 & 312 & 0.72 & 0.78 & 0.86 & 0.79 & 20 & 63 \\
\hline & & & 6 & 293 & 0.72 & 0.76 & 0.85 & 0.79 & 23 & 59 \\
\hline & & & 11 & 288 & 0.72 & 0.75 & 0.84 & 0.79 & 26 & 58 \\
\hline & & & 14 & 282 & 0.72 & 0.74 & 0.84 & 0.79 & 29 & 57 \\
\hline & & & 0 & 552 & 1.00 & 0.78 & 0.90 & 0.79 & 37 & 112 \\
\hline & & & 0 & 812 & 1.00 & 0.78 & 0.91 & 0.79 & 44 & 165 \\
\hline & & & 0 & 1080 & 1.00 & $\begin{array}{l}0.78 \\
\end{array}$ & 0.92 & 0.79 & 51 & 219 \\
\hline & & & 0 & 1381 & 1.00 & 0.78 & 0.93 & 0.79 & 59 & 281 \\
\hline & & & 0 & 1468 & 0.97 & 0.78 & 0.92 & 0.79 & 66 & 298 \\
\hline & & & 0 & 1465 & 0.92 & 0.78 & 0.91 & 0.79 & 73 & 298 \\
\hline & & & 0 & 1453 & 0.86 & 0.78 & 0.90 & 0.79 & 80 & 295 \\
\hline & & & 3 & 1435 & 0.86 & 0.78 & 0.89 & 0.79 & 87 & 292 \\
\hline & & & 17 & 1438 & 0.83 & 0.77 & 0.88 & 0.79 & 96 & 292 \\
\hline & & & 54 & 1404 & 0.81 & 0.75 & 0.87 & 0.79 & 110 & 285 \\
\hline & & & 105 & $\begin{array}{c}1481 \\
2\end{array}$ & \multicolumn{6}{|c|}{ المجموع } \\
\hline & & & 3 & 411 & \multicolumn{6}{|c|}{ المعدل لموسم نمو كامل } \\
\hline
\end{tabular}


محمود: نمذجة تأثير تناسق الإرواء على كفاءة وكفاية وتناست الإرواء المفيد والإنتاجية

الجدول (9) : نتائج الأنموذج الحاسوبي للنمط الثالث ولزمن تشغيلي 100\%

\begin{tabular}{|c|c|c|c|c|c|c|c|c|c|c|}
\hline استخدام & الإقتقي & الإنتاجة & 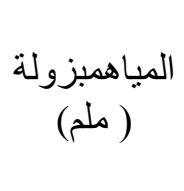 & كميةالمياه & الإرواءة & الإرواءة & التإسقاء & الإرواء & اليوم & التشغيل \\
\hline \multirow{12}{*}{0.13} & \multirow{12}{*}{732} & \multirow{12}{*}{0.91} & 121 & 710 & 1.00 & 0.59 & 0.93 & 0.66 & 7 & 157 \\
\hline & & & 185 & 788 & 0.94 & 0.54 & 0.88 & 0.66 & 14 & 174 \\
\hline & & & 201 & 727 & 0.89 & 0.52 & 0.86 & 0.66 & 20 & 161 \\
\hline & & & 204 & 740 & 0.89 & 0.52 & 0.86 & 0.66 & 26 & 164 \\
\hline & & & 283 & 1274 & 1.00 & 0.55 & 0.90 & 0.66 & 36 & 282 \\
\hline & & & 507 & 2455 & 1.00 & 0.57 & 0.90 & 0.66 & 48 & 543 \\
\hline & & & 756 & 3621 & 1.00 & 0.56 & 0.90 & 0.66 & 61 & 801 \\
\hline & & & 867 & 3527 & 0.94 & 0.54 & 0.89 & 0.66 & 73 & 780 \\
\hline & & & 899 & 3638 & 0.94 & 0.54 & 0.88 & 0.66 & 86 & 805 \\
\hline & & & 956 & 3546 & 0.94 & 0.52 & 0.87 & 0.66 & 105 & 785 \\
\hline & & & 4978 & 21025 & \multicolumn{6}{|c|}{ المجموع } \\
\hline & & & 138 & 584 & \multicolumn{6}{|c|}{ المعدل لموسم نمو كامل } \\
\hline
\end{tabular}

الجدول (10) : نتائج الأنموذج الحاسوبي للنمط الثالث ولزمن تشغيلي 80\%

\begin{tabular}{|c|c|c|c|c|c|c|c|c|c|c|}
\hline استخداءم & الحقيقي & الإنتاج & مبزولة المباه & كمية|الم & $\begin{array}{c}\text { كفاية } \\
\text { الإروا } \\
\text { كاية }\end{array}$ & الإرواء كفاءة & الإرواء & الإرواء & 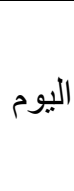 & التشغيل \\
\hline \multirow{13}{*}{0.14} & \multirow{13}{*}{715} & \multirow{13}{*}{0.89} & 31 & 568 & 1.00 & 0.67 & 0.92 & 0.66 & 7 & 126 \\
\hline & & & 98 & 595 & 0.89 & 0.59 & 0.86 & 0.66 & 13 & 132 \\
\hline & & & 113 & 613 & 0.81 & 0.58 & 0.85 & 0.66 & 19 & 136 \\
\hline & & & 125 & 568 & 0.81 & 0.55 & 0.84 & 0.66 & 24 & 126 \\
\hline & & & 126 & 564 & 0.78 & 0.55 & 0.84 & 0.66 & 29 & 125 \\
\hline & & & 152 & 1340 & 1.00 & 0.63 & 0.88 & 0.66 & 40 & 296 \\
\hline & & & 242 & 2176 & 1.00 & 0.63 & 0.89 & 0.66 & 51 & 481 \\
\hline & & & 364 & 2889 & 1.00 & 0.62 & 0.88 & 0.66 & 63 & 639 \\
\hline & & & 477 & 2820 & 0.94 & 0.59 & 0.87 & 0.66 & 74 & 624 \\
\hline & & & 492 & 2898 & 0.89 & 0.59 & 0.86 & 0.66 & 86 & 641 \\
\hline & & & 555 & 2828 & 0.81 & 0.57 & 0.85 & 0.66 & 102 & 626 \\
\hline & & & 2775 & 17859 & \multicolumn{6}{|c|}{ المجموع } \\
\hline & & & 77 & 496 & \multicolumn{6}{|c|}{ المعدل لموسم نمو كامل } \\
\hline
\end{tabular}


الجدول (11) : نتائج الأنموذج الحاسوبي للنمط الثالث ولزمن تشغيلي 60\%

\begin{tabular}{|c|c|c|c|c|c|c|c|c|c|c|}
\hline كيتخدام & الإقإتتاج & الإنتاج & مبزولة المياه & كمية المياه & الإرواء كفاءة & الإرواء & التاسقق & الإرواء & اليوم اليوم & (دقتشغيل \\
\hline \multirow{14}{*}{0.17} & \multirow{14}{*}{688} & \multirow{14}{*}{0.86} & 0 & 426 & 1.00 & 0.71 & 0.92 & 0.66 & 7 & 94 \\
\hline & & & 23 & 458 & 0.81 & 0.68 & 0.86 & 0.66 & 12 & 101 \\
\hline & & & 41 & 455 & 0.75 & 0.65 & 0.83 & 0.66 & 17 & 101 \\
\hline & & & 52 & 455 & 0.72 & 0.63 & 0.82 & 0.66 & 22 & 101 \\
\hline & & & 51 & 461 & 0.72 & 0.63 & 0.82 & 0.66 & 27 & 102 \\
\hline & & & 22 & 779 & 0.94 & 0.69 & 0.86 & 0.66 & 36 & 172 \\
\hline & & & 33 & 1344 & 1.00 & 0.69 & 0.87 & 0.66 & 46 & 297 \\
\hline & & & 45 & 1996 & 1.00 & 0.70 & 0.88 & 0.66 & 57 & 442 \\
\hline & & & 127 & 2193 & 0.89 & 0.67 & 0.86 & 0.66 & 67 & 485 \\
\hline & & & 170 & 2156 & 0.81 & 0.66 & 0.85 & 0.66 & 77 & 477 \\
\hline & & & 205 & 2140 & 0.81 & 0.64 & 0.84 & 0.66 & 87 & 474 \\
\hline & & & 255 & 2135 & 0.78 & 0.63 & 0.83 & 0.66 & 101 & 472 \\
\hline & & & 1024 & 14998 & \multicolumn{6}{|c|}{ المجموع } \\
\hline & & & 28 & 417 & \multicolumn{6}{|c|}{ المعدل لموسم نمو كامل } \\
\hline
\end{tabular}

الجدول (12) : نتائج الأنموذج الحاسوبي للنمط النالث ولزمن تشغيلي 40\%

\begin{tabular}{|c|c|c|c|c|c|c|c|c|c|c|}
\hline الرتخدام & الحقيقي & الإنتاج & لزيلة المياهد & كميةالم & الإرواءة & الإرواء كفاءة & الإرواء & الإرواء & اليوم & زالتشغيل \\
\hline \multirow{19}{*}{0.18} & \multirow{19}{*}{673} & \multirow{19}{*}{0.84} & 0 & 284 & 1.00 & 0.71 & 0.94 & 0.66 & 7 & 63 \\
\hline & & & 0 & 290 & 0.81 & 0.71 & 0.89 & 0.66 & 10 & 64 \\
\hline & & & 2 & 282 & 0.72 & 0.71 & 0.85 & 0.66 & 13 & 62 \\
\hline & & & 7 & 304 & 0.67 & 0.70 & 0.82 & 0.66 & 17 & 67 \\
\hline & & & 23 & 285 & 0.67 & 0.66 & 0.81 & 0.66 & 20 & 63 \\
\hline & & & 12 & 306 & 0.67 & 0.68 & 0.81 & 0.66 & 24 & 68 \\
\hline & & & 26 & 286 & 0.67 & 0.65 & 0.80 & 0.66 & 27 & 63 \\
\hline & & & 24 & 281 & 0.67 & 0.65 & 0.80 & 0.66 & 30 & 62 \\
\hline & & & 0 & 628 & 0.81 & 0.71 & 0.87 & 0.66 & 39 & 139 \\
\hline & & & 0 & 951 & 0.89 & 0.71 & 0.89 & 0.66 & 47 & 210 \\
\hline & & & 0 & 1246 & 0.89 & 0.71 & 0.89 & 0.66 & 55 & 276 \\
\hline & & & 0 & 1475 & 0.81 & 0.71 & 0.88 & 0.66 & 63 & 326 \\
\hline & & & 13 & 1465 & 0.75 & 0.71 & 0.87 & 0.66 & 70 & 324 \\
\hline & & & 49 & 1426 & 0.72 & 0.69 & 0.85 & 0.66 & 77 & 315 \\
\hline & & & 67 & 1414 & 0.72 & 0.68 & 0.85 & 0.66 & 84 & 313 \\
\hline & & & 77 & 1422 & 0.72 & 0.67 & 0.84 & 0.66 & 92 & 315 \\
\hline & & & 111 & 1413 & 0.72 & 0.66 & 0.83 & 0.66 & 104 & 313 \\
\hline & & & 410 & 13758 & \multicolumn{6}{|c|}{ المجموع } \\
\hline & & & 11 & 382 & \multicolumn{6}{|c|}{ المعدل لموسم نمو كامل } \\
\hline
\end{tabular}


محمود: نمذجة تأثير تناسق الإرواء على كفاءة وكفاية وتناست الإرواء المفيد والإنتاجية

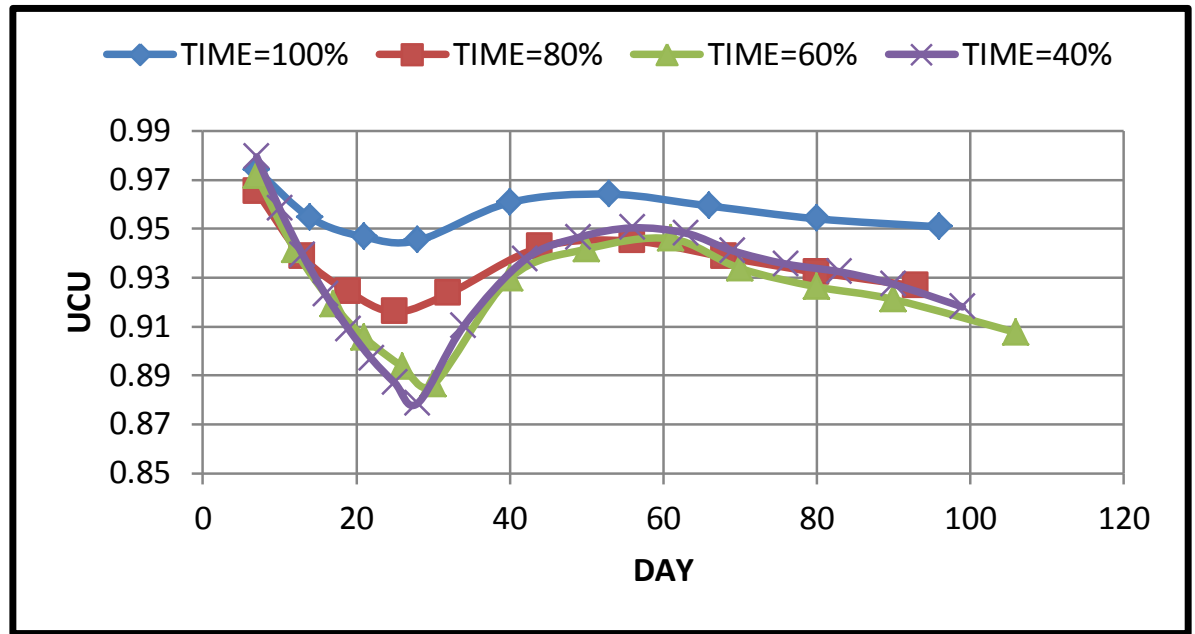

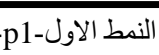
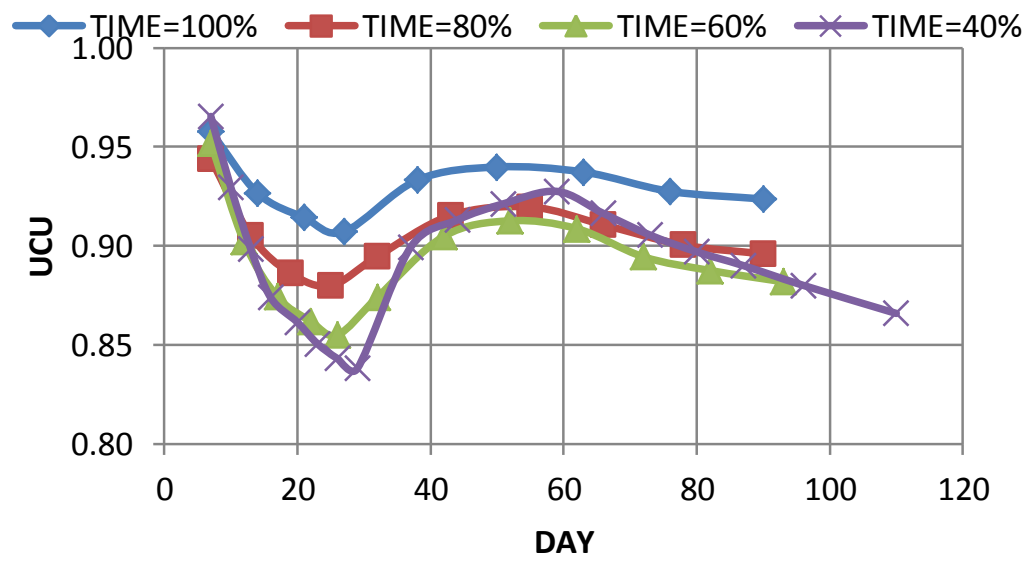

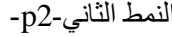

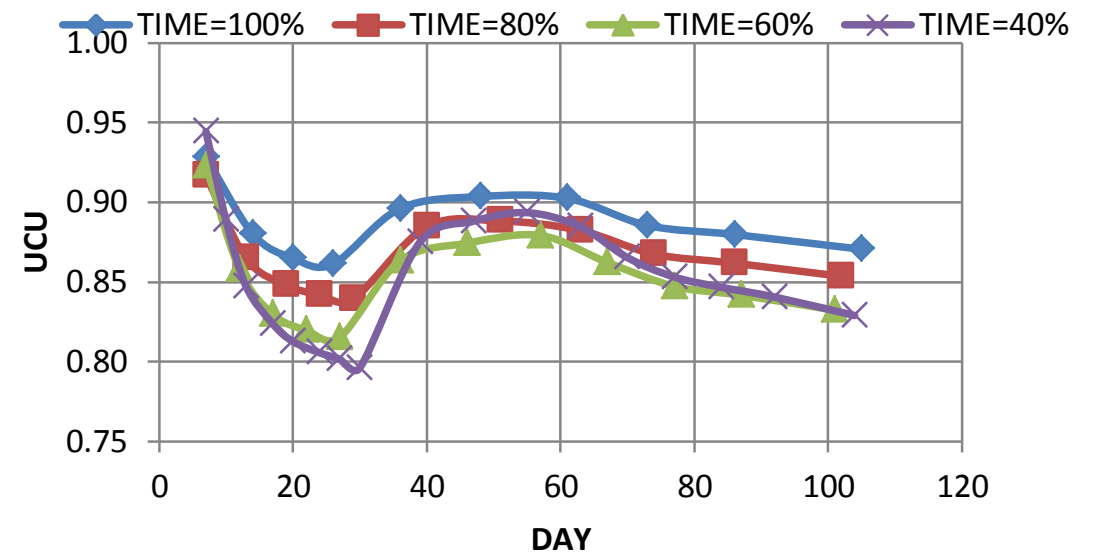

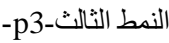

الثكل (1): العلاقة بين تناسق الإرواء المفيد خلال موسم نمو المحصول و لأزمنة تشغيل مختلفة 


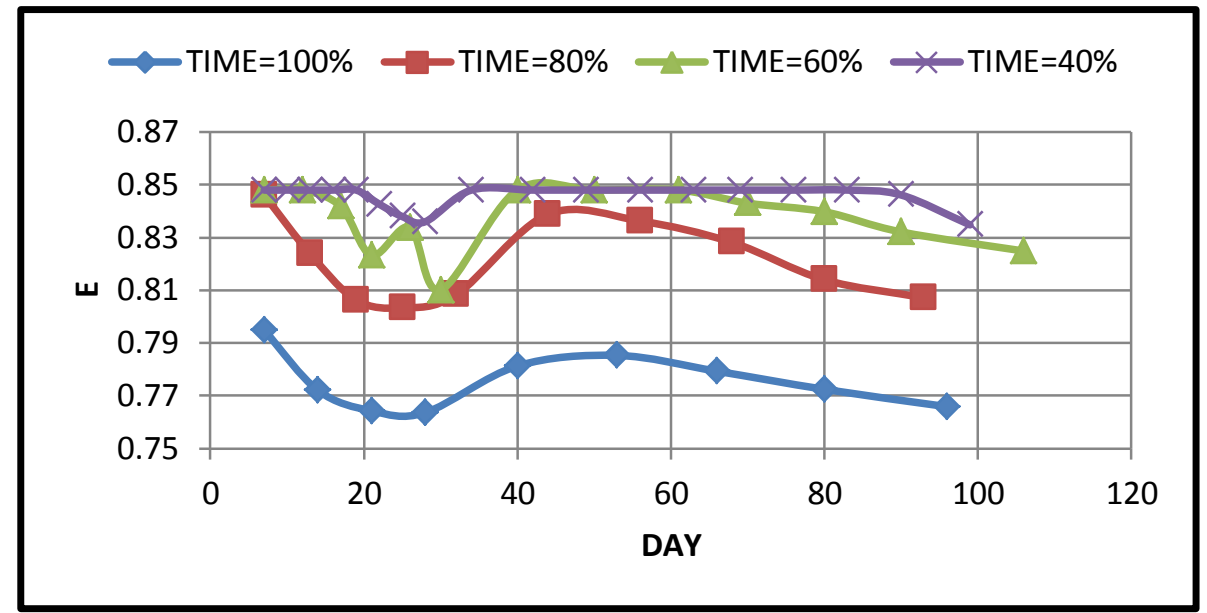

النمط الاول-p1-
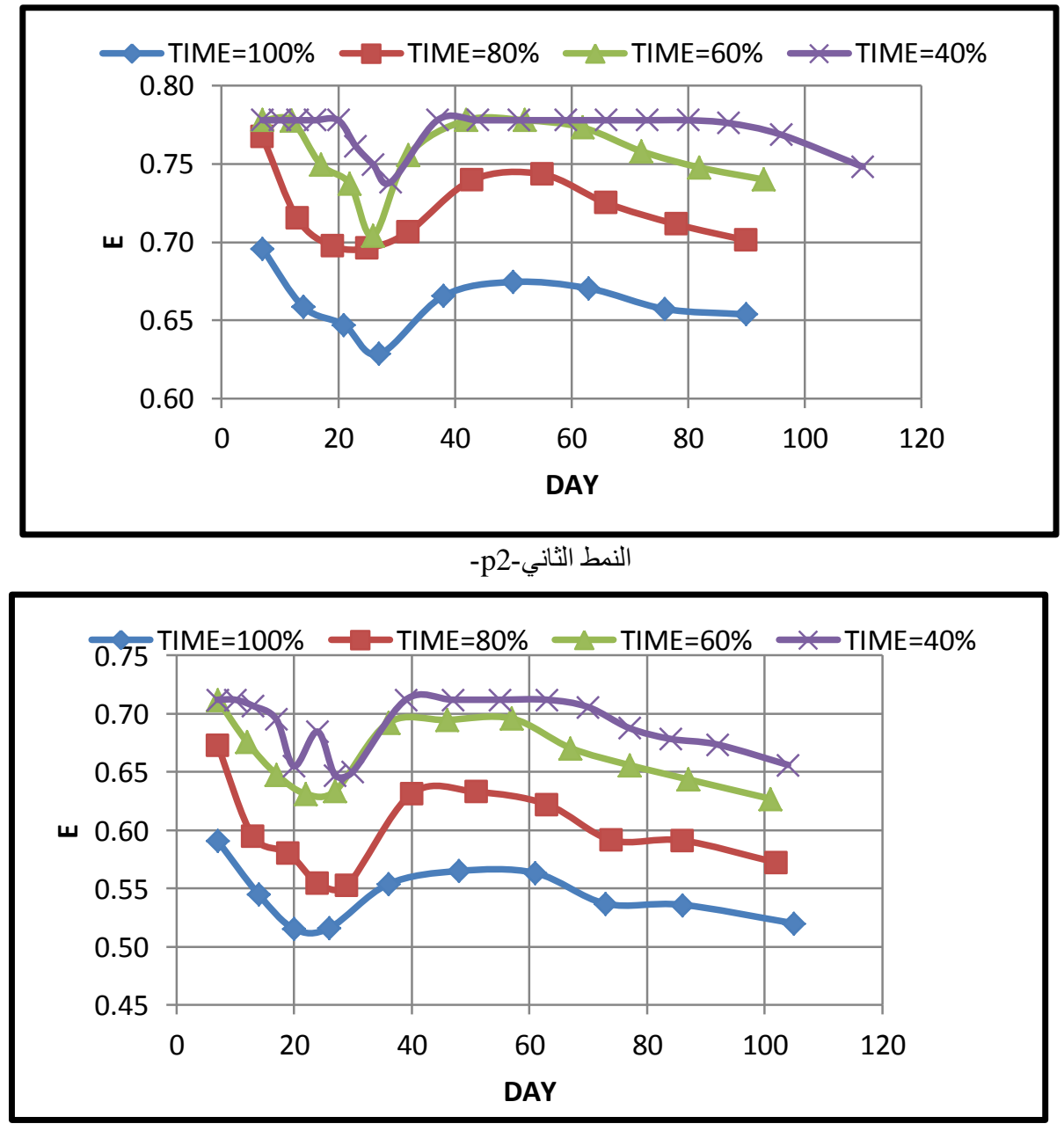

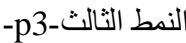

الثكل (2): العلاقة بين كفاءة الإرواء خلال موسم نمو المحصول و لأزمنة تتغيل مختلفة 
محمود: نمذجة تأثير تناسق الإرواء على كفاءة وكفاية وتناست الإرواء المفيد والإنتاجية

$\longrightarrow$ TIME=100\% -TIME=80\% $\leftarrow$ TIME=60\% $\leftarrow$ TIME=40\%

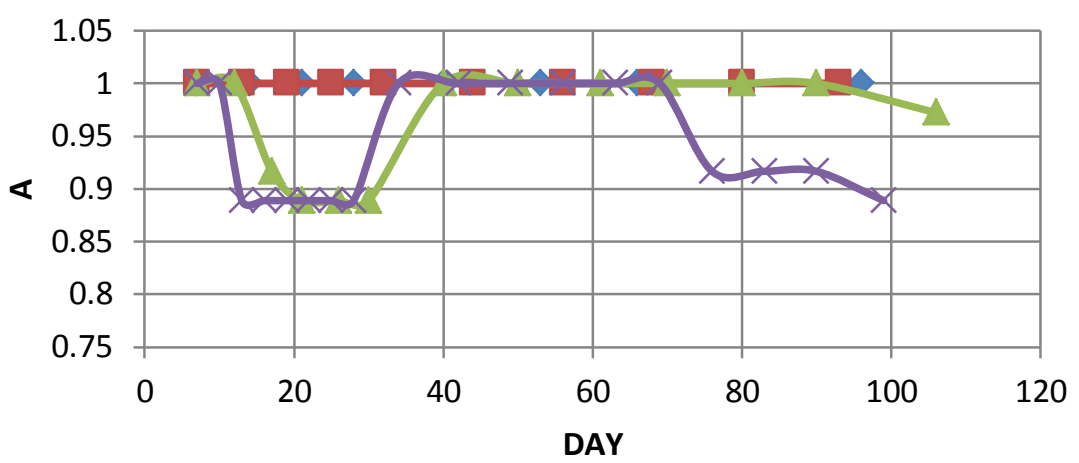

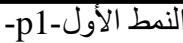

$\sim$ TIME=100\% - TIME=80\% $\longrightarrow$ TIME=60\% $\leftarrow$ TIME $=40 \%$

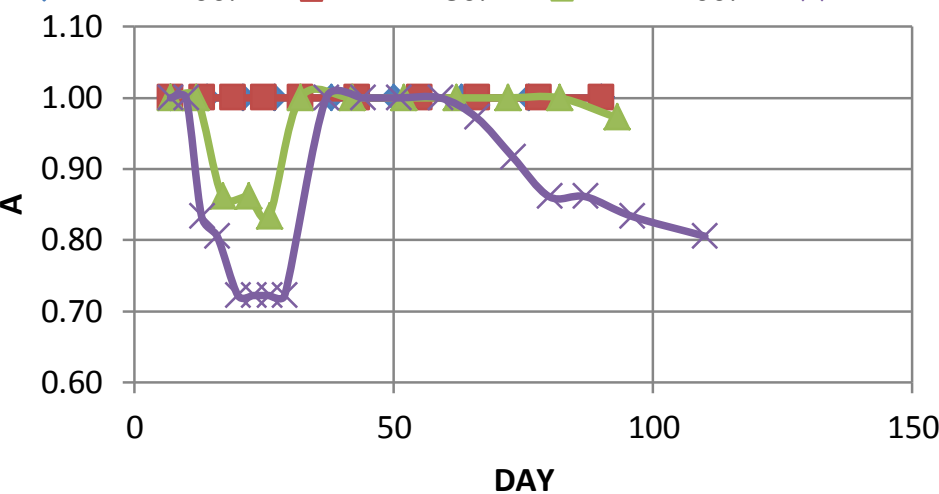

النمط الثناني -p2-

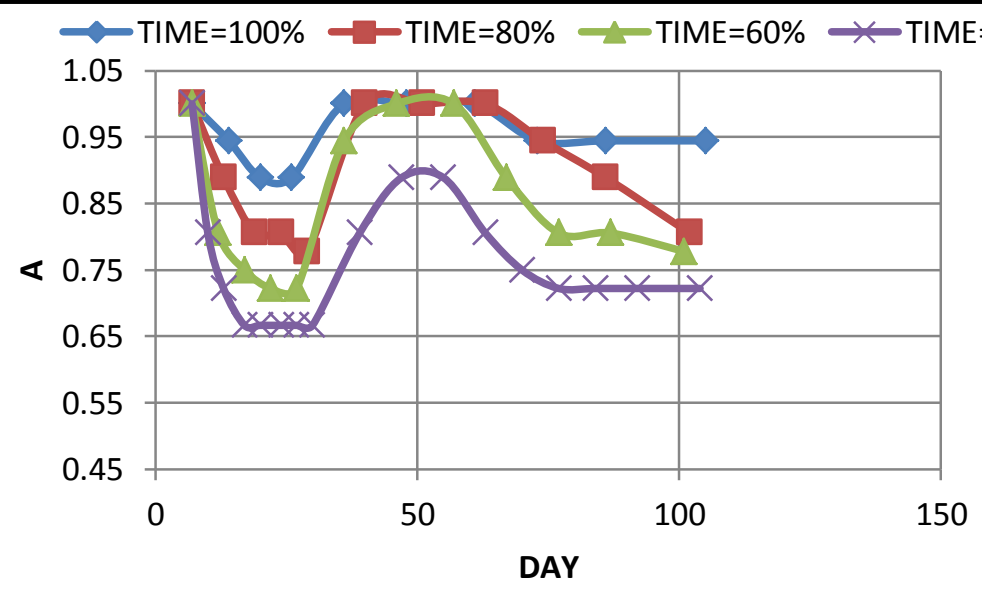

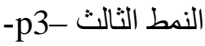

الثكل (3): العلاقة بين كفاية الإرو اء خلال موسم نمو المحصول و لأزمنة تشغيل مختلفة 


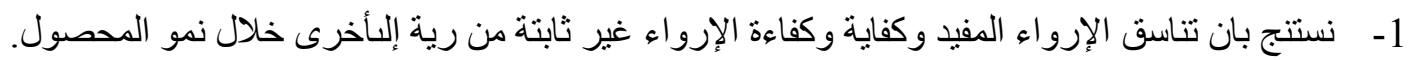

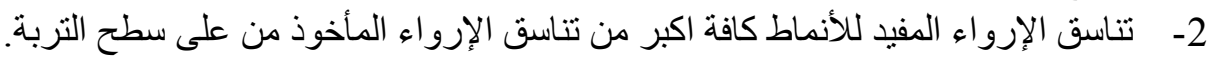

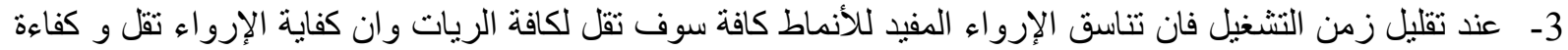

الإرواء تزداد نتيجة قلة المياه الضائعة.

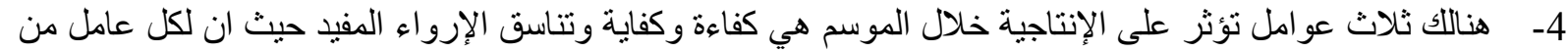

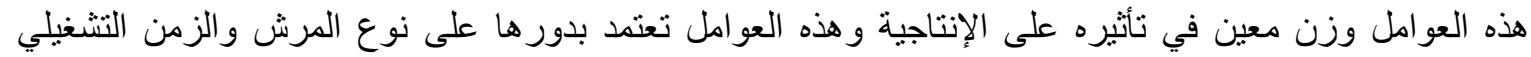

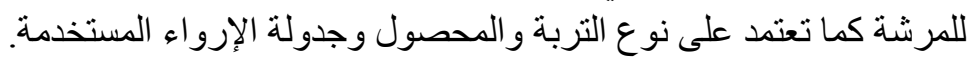

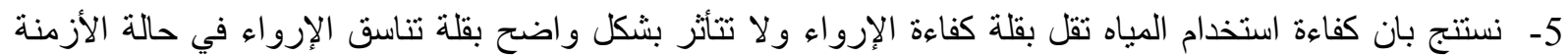

التشغيلية القلبلة.

1آل أميادر اغا، عصام عبد القادر (2001)." نأثير بعض العو امل في في انتظام توزيع الماء و إنتاجية الذرة الصفر اء في نظسام

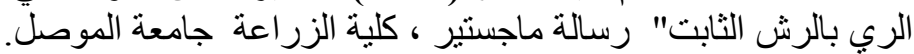

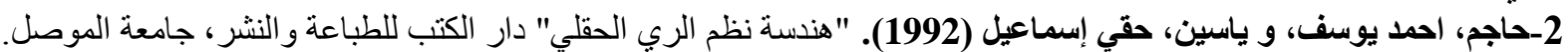

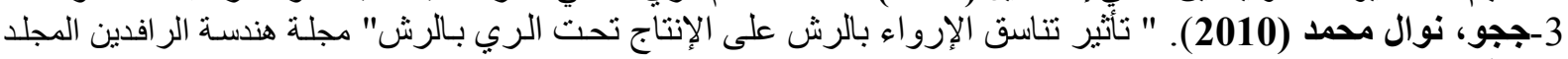
2 العدي 18

4ياسين، حقي إسماعيل (1994)." تأثير الريح على تتاسق الإرواء لنظم الرش الثابتة" مجلة هندسة الر افدين المجلد 2 ،العدد 2 .

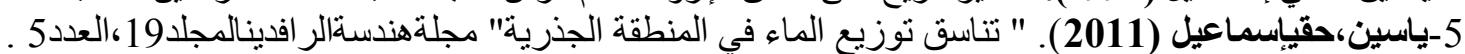

6-Allen, R. G., Pereira, L. S., Raes, D. and Smith, M., (1998). " Crop EvapotranspirationGuidelines for Computing Crop Water Requirements ". FAO Irrigation and Drainage paper No.56, Rome, Italy.

7-Li, J.and Rao, M.(1999)."Crop yield as affected by uniformity of sprinkler Irrigation system" .Agricultural Engineering International ,The CIGR,Journal of scientific research and development ,Manuscript VOL.3,China.

8- Solomon, K.H. (1990) ."Sprinkler irrigation uniformity". Irrigation note, California state University , August, 1990

9-YASIN, H. I . (1984) " Effect of Riser Height And Pressure on Uniformity of Water Distribution Under Stationary Sprinkler Systems” M.Sc. Thsis, University of Mosul .

تم اجراء البحث في كلية ألهندسة = جامعة ألموصل 